Printed in Great Britain

\title{
Ein neuer Beweis eines Satzes von Magnus
}

\author{
BY U. STAMMBACH \\ Eidgen. Technische Hochschule, Zürich, Switzerland
}

(Received 15 December 1966)

Summary. The author provides a new and very simple proof of the following theorem of Magnus (2):

THEOREM. Let the group $G$ have both a presentation with $n+r$ generators and $r$ relations also a presentation with $n$ generators. Then $G$ is the free group on $n$ generators.

Der Inhalt dieser Note besteht in einem neuen, sehr einfachen Beweis des folgenden Satzes von Magnus (2):

Satz. Besitzt die Gruppe G eine Präsentierung mit $n+r$ Erzeugenden undr Relationen und besitzt sie auch eine Präsentierung mit $n$ Erzeugenden, so ist $G$ die freie Gruppe mit $n$ Erzeugenden.

Unser Beweis verwendet eine exakte Sequenz aus der Homologietheorie der Gruppen, die von Stallings(3) und dem Verfasser (4) benützt worden ist, um Beziehungen zwischen den Homologiegruppen und den Zentralreihen einer Gruppe herzuleiten.

Wir beginnen mit einem einfachen Lemma, das den Rang von abelschen Gruppen betrifft. Ist $A$ eine abelsche Gruppe, so verstehen wir unter ihrem $\operatorname{Rang} \operatorname{Rg} A \operatorname{die}$ Dimension des Vektorraumes $\mathbf{Q} \otimes A$. Dabei ist $\mathbf{Q}$ der Körper der rationalen Zahlen. Das Tensorprodukt ist über dem Ring der ganzen Zahlen zu nehmen.

LEmma. Es sei $0 \rightarrow A \rightarrow B \rightarrow C \rightarrow 0$ eine exakte Sequenz von endlich erzeugten abelschen Gruppen. Dann gilt $\operatorname{Rg} A+\operatorname{Rg} C=\operatorname{Rg} B$.

Beweis. Man tensoriere die exakte Sequenz mit $\mathbf{Q}$ und benütze, dass Tor $(\mathbf{Q}, C)=0$ ist.

Beweis des Satzes. Es sei $F / R \cong G$ eine Präsentierung der Gruppe $G$ durch die freie Gruppe $F$. Wir entnehmen dann aus (3) oder (4) folgende exakte Sequenz von abelschen Gruppen

(I) $0 \rightarrow H_{2}(G) \rightarrow R /[F, R] \rightarrow H_{1}(F) \rightarrow H_{1}(G) \rightarrow 0$.

$[F, R]$ bezeichnet dabei die Untergruppe von $F$, die von allen Kommutatoren der Form $y x y^{-1} x^{-1}$ mit $y \in F$ und $x \in R$ erzeugt wird. $H_{i}(G)$ ist die $i-$ te Homologiegruppe von $G$ mit Koeffizienten in $\mathbf{Z}$, der additiven Gruppe der ganzen Zahlen; insbesondere ist $H_{1}(G)$ isomorph zur 'abelsch gemachten' Gruppe $G /[G, G]$. (Die Sequenz (I) lässt sich auch leicht mit Hilfe der Hopfschen Formel für die zweite ganzzahlige Homologiegruppe $H_{2}(G) \cong([F, F] \cap R) /[F, R]$ beweisen; und zwar benützen wir im folgenden nur die Tatsache, dass die durch die Formel $([F, F] \cap R) /[F, R]$ gegebene Gruppe von der gewählten Präsentierung unabhängig ist.)

Wir betrachten nun zuerst die Präsentierung unserer Gruppe $G$ durch $n+r$ Erzeugende und $r$ Relationen: $G \cong F(n+r) / R(r)$. Wenn wir die Gruppe

$$
R(r) /[F(n+r), R(r)]
$$


mit $A$ bezeichnen und unsere exakte Sequenz (I) in der Mitte aufspalten, so erhalten wir die beiden folgenden kurzen exakten Sequenzen:

$$
\begin{gathered}
\text { (II) } 0 \rightarrow H_{2}(G) \rightarrow A \rightarrow A^{\prime} \rightarrow 0, \\
\text { (III) } 0 \rightarrow A^{\prime} \rightarrow Z^{n+r} \rightarrow G /[G, G] \rightarrow 0 .
\end{gathered}
$$

$A^{\prime}$ bezeichnet hier also den Kern des Homomorphismus $H_{1}(F) \cong Z^{n+r} \rightarrow G /[G, G]$ bzw. das Bild der vorhergehenden Abbildung. Da $G /[G, G]$ als Quotient von $G$ durch $n$ Elemente erzeugt wird, also einen Rang $\leqslant n$ besitzt, folgt mit unserem Lemma

$$
\operatorname{Rg} A^{\prime} \geqslant r .
$$

$A^{\prime}$ ist als Untergruppe einer freien abelschen Gruppe selbst frei abelsch; folglich zerfällt die Sequenz (II), d.h. $A^{\prime}$ ist direkter Summand in $A$. Wie man leicht feststellt wird diese Gruppe $A=R(r) /[F(n+r), R(r)]$ durch $r$ Elemente, nämlich durch die Relationen erzeugt. Als direkter Summand in $A$ wird damit auch $A^{\prime}$ durch $r$ Elemente erzeugt. Mit (1) ergibt sich somit

$$
A^{\prime} \cong \mathbf{Z}^{r} \text {. }
$$

Da die Gruppe $A$ durch $r$ Elemente erzeugt wird und $A^{\prime} \cong \mathbf{Z}^{r}$ als direkten Summanden enthält, folgt, dass das direkte Komplement von $A^{\prime}$ in $A$ gleich 0 ist:

$$
H_{2}(G)=0 .
$$

Eine weitere Anwendung unseres Lemmas auf die Sequenz (III) ergibt mit (2) $\operatorname{Rg} G /[G, G]=n$. Da die Gruppe $G /[G, G]$ durch $n$ Elemente erzeugt wird, ist also

$$
H_{1}(G) \cong G /[G, G] \cong \mathbf{Z}^{n}
$$

Betrachten wit nun weiter die Präsentierung $F / R$ von $G$ durch $n$ Elemente und die dazugehörige exakte Sequenz (I), so erhalten wir mit (3) und (4)

$$
0 \rightarrow 0 \rightarrow R /[F, R] \rightarrow \mathbf{Z}^{n} \rightarrow \mathbf{Z}^{n} \rightarrow 0 .
$$

Es folgt $R /[F, R]=0$. Man beweist nun leicht mit Induktion, dass ein Normalteiler $R$ einer Gruppe $F$ mit der Eigenschaft $R=[F, R]$ in jedem Glied $F_{k}$ der absteigenden Zentralreihe von $F$ enthalten ist. Da nun für eine freie Gruppe $F$ der Durchschnitt der $F_{k}$ nach Magnus (1) nur aus dem Neutralelement $e$ von $F$ besteht, ist $R=\{e\}$ und $F \cong G$.

\section{LITERATUR}

(1) Magnus, W. Beziehungen zwischen Gruppen und Idealen in einem speziellen Ring. Math. Ann. 111 (1935), 259-280.

(2) Magnus W. Ueber freie Faktorgruppen und freie Untergruppen gegebener Gruppen. Monatsh. Math. 47 (1939), 307-313.

(3) Staltings, J. Homology and central series of groups. J. Algebra 2 (1965), 170-181.

(4) Stammaach, U. Anwendungen der Homologietheorie der Gruppen auf Zentralreihen und auf Invarianten von Präsentierungen. Math. Z. 94 (1966), 157-177. 